\title{
Validación psicométrica de la versión breve del Inventario de Características Institucionales Estresoras
}

\section{Psychometric validation of the short version of the Inventory of Stressor-related Institutional Characteristics} \section{(1) PSicOCERTE}

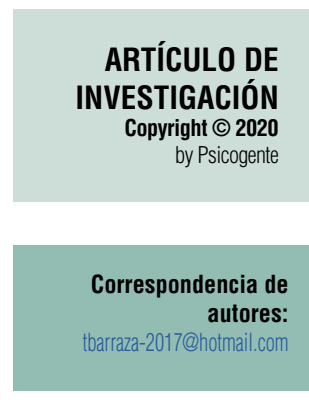

Recibido: 06/03/19 Aceptado: $14 / 08 / 19$ Publicado: $01 / 01 / 20$

\author{
Arturo Barraza-Macías iD \\ Universidad Pedagógica de Durango, Durango, México.
}

\section{Resumen}

Objetivo: Realizar la validación psicométrica del Inventario de Características Institucionales Estresoras.

Método: Estudio instrumental desarrollado a través de la aplicación del Inventario de Características Institucionales Estresoras a 300 alumnos de la Universidad Pedagógica de Durango, en Durango, México; de estos alumnos el $32 \%$ son del sexo masculino y el 68\% del sexo femenino, la edad mínima es de 19 años y la máxima de 67 años, siendo el promedio 29 años de edad, y el 75\% cursaba en ese momento la licenciatura y el $25 \%$ restante la maestría o doctorado.

Resultados: Se construyó una versión breve del inventario, la cual obtuvo un nivel de confiabilidad de .71 en alfa de Cronbach; en el análisis de consistencia interna todos los ítems correlacionaron positivamente (con un nivel de significación de $\mathrm{p}<$.001) con el puntaje global obtenido por cada encuestado; el análisis factorial exploratorio reporta dos componentes que explican $40 \%$ de la varianza total y el análisis factorial confirmatorio muestra un buen ajuste a dos factores $(\chi 2=16,70 ; R M S E A=0,000 ; \chi 2 / d f=0,49)$.

Conclusión: Las características institucionales generadoras del estrés, en las instituciones escolares, es una de las áreas del campo de estudio del estrés menos investigada; una de las razones centrales es la falta de instrumentos que permitan medir cómo diversas variables situacionales influyen en la valoración que el sujeto hace de las demandas para considerarlas estresores. En ese sentido, esta versión breve del ICIE se vuelve una opción válida para este tipo de estudios.

Palabras clave: estrés académico; validación psicométrica; instituciones educativas; demandas estresantes.

Abstract

Objective: Perform the psychometric validation of the Inventory of Stressor Institutional Characteristics.

Method: Instrumental study developed through the application of the Inventory of Stressor Institutional Characteristics to 300 students of the Pedagogical University of Durango, Durango, Mexico; of these students $32 \%$ are male and $68 \%$ female, the minimum age is 19 years and the maximum of 67 , with the average being 29 years of age, and $75 \%$ were studying at the time the degree and the remaining $25 \%$ the masters or doctorate.

Results: A short version of the inventory was built, which obtained a reliability level of .71 in Cronbach's alpha; in the analysis of internal consistency, all the items correlated positively (with a level of significance of $p<.001$ ) with the overall score obtained by each respondent; exploratory factor analysis reports two components that explain $40 \%$ of the total variance and the confirmatory factor analysis shows a good adjustment to two factors ( $\chi 2=16.70, R M S E A=0.000, \chi 2 / d f=0.49$ ).

Conclusion: Institutional characteristics that generate stress, in school institutions, is one of the least researched areas; one of the main reasons is the lack of instruments that allow us to measure how various situational variables influence the assessment that the subject makes of the demands to consider them stressors. In this sense, this short version of the ICIE becomes a valid option for this type of studies.

Key words: academic stress; psychometric validation; educational institutions; stressful demands.

Cómo citar este artículo (APA): 


\section{INTRODUCCIÓN}

Las instituciones educativas, en lo general, y sus docentes, en lo particular, suelen exigirles a sus alumnos la realización de una serie de labores escolares como parte de los procesos de enseñanza y aprendizaje que desarrollan en sus aulas; ante estas exigencias, el alumno realiza una valoración cognitiva de las mismas en función de los recursos que posee para enfrentar exitosamente su ejecución. Cuando la demanda sobrepasa sus recursos para enfrentarla, le es asignado un significado relacional que la constituye en un estresor que desencadena el proceso adaptativo que denominamos estrés.

Los estudios al respecto se han enfocado a estudiar su prevalencia (Álvarez, Gallegos \& Herrera, 2018; Macbani et al., 2018; Quito, Tamayo, Buñay \& Neira, 2017; Rodríguez, Arenal, Vega \& Blanco, 2018; Velásquez-Muñoz, Cornejo-Aravena, Bustamante-Toncio \& Mella-Guzmán, 2018) o su relación con otras variables individuales, o propias del sujeto, como sería el caso del rendimiento académico o escolar (Fernández de Castro \& Luévano, 2018; Vidal, Muntaner \& Palau, 2018), la calidad de sueño (You-Wei, Rong-Mao, Yan-Kui \& Ming-Yan, 2018), la depresión (Barker, Howard, Villemaire-Krajden \& Galambos, 2018; Emiro, et al., 2018; Sakellari, et al., 2017), los perfiles de regulación emocional (González, Souto-Gestal \& Fernández, 2017), la persistencia en el aprendizaje (Won, 2018), la autoeficacia (Andretta, McKay \& Byrne, 2018), la orientación de las metas (González, Franco, Souto-Gestal \& González, 2017), la procrastinación (Anjali, 2018) y la autorregulación (Hamizah, Alavi, Abolghasem \& Ahmadi, 2018), por mencionar solamente a algunas.

No obstante esta multiplicidad y variedad de estudios, es necesario reconocer que el proceso de valoración cognitiva, que da lugar a la aparición del estrés y que es planteado por Lazarus y Folkman (1986) y Lazarus (2000), presenta como antecedente un conjunto de condiciones ambientales y características individuales que entran en transacción para otorgarle a la demanda ese significado relacional; sin embargo, en la mayoría de los estudios, sobre la generación del estrés, los investigadores se han centrado en el individuo (vid supra), descuidando el contexto como parte interviniente en este proceso. A este respecto hay que reconocer que si bien la mayoría de las investigaciones sobre el origen del estrés se han centrado en el estudio del papel que juegan determinados rasgos de personalidad de los propios sujetos, existe otra perspectiva denominada contextualista (Aldwin \& Revenson, 1987; Lazarus \& Folkman, 1986; Moss, 1995, 2010, 2018; Ribes, 1990) que busca asignarle un papel más protagónico a la naturaleza de la situación, esto es, a las características contextuales; particularmente de interés para esta investigación son los 
aportes que realizan Lazarus y Folkman (1986) y Ribes (1990): los primeros analizan los factores situacionales que influyen en la valoración, como sería el caso de la novedad, incertidumbre, inminencia, duración y ambigüedad de la situación, mientras que el segundo plantea tres situaciones contingenciales relacionadas con el estrés: ambigüedad, incertidumbre e incontrolabilidad.

La existencia de esta perspectiva contextualista, en el estudio del estrés, ha sido nominalista y en realidad no ha aportado un gran acervo empírico e hipótesis teóricas al respecto en los últimos años. Los pocos estudios en esta línea presentan dos orientaciones básicas: su relación con la cultura nacional o su relación con contextos curriculares. En la primera línea los autores estudian los rasgos de las culturas nacionales como potenciales características explicativas del estrés (Barraza \& León, 2015; González, 2008). Barraza y León (2015) se plantearon como objetivo central determinar los estresores que se presentaban con mayor y menor frecuencia entre los educandos de las carreras de educación de una universidad mexicana y una costarricense, obteniendo como resultado que existe similitud en las dos universidades en la frecuencia con que se presentan los estresores académicos; por su parte González (2008), en su investigación cualitativa, se orientó a describir la experiencia del estrés académico en estudiantes peruanos y suecos que cursaban el segundo año de la carrera de Educación, pudiendo afirmar, a partir de sus resultados, que los estudiantes de ambos países coinciden en las situaciones académicas que consideran estresantes, en su percepción negativa de los efectos del estrés en su desempeño, salud y estado de ánimo, y en su interés en un curso sobre afrontamiento.

En la segunda línea los autores exploran si las condiciones derivadas de la operación de un determinado plan de estudios influyen en la generación de estresores (Arce, Fariña, Novo \& Seijo, 2012; Harajyoti, Dipankar, Lipika \& Nabanita, 2012; Shashank, Richa \& Prakash, 2011). Arce et al. (2012) desarrollaron un estudio con 200 estudiantes de Fisioterapia, 110 de ellos estudiaban en el sistema de créditos tradicional, y los otros 100, en el Sistema de Créditos Europeos, obteniendo como resultado que los estudiantes del sistema de créditos europeos manifestaron mayores niveles de ira y pensamientos negativos; mientras que Shashank et al. (2011) llevaron a cabo un estudio comparativo sobre el estrés en estudiantes de Medicina, Ingeniería y Enfermería obteniendo como resultado que el estrés reportado era mayor en los alumnos de Ingeniería y Medicina; por su parte Harajyoti et al. (2012) realizaron un estudio comparativo sobre el estrés, y los factores que contribuyen a su aparición, entre estudiantes de Licenciatura y de Postgrado, obteniendo como resultado que los estudiantes de Postgrado fueron más susceptibles al estrés que los estudiantes de Licenciatura. 
En términos de Lazarus (2000), la primera orientación busca las causas distales, mientras que la segunda busca las causas proximales. Los resultados parecen indicar la supremacía de la orientación proximal, sin embargo, estas investigaciones no brindan suficiente información sobre qué características, rasgos o aspectos de la operación de un determinado plan de estudios pueden afectar la presencia de mayor estrés; en este punto solamente Arce et al. (2012) y Harajyoti et al. (2012) enuncian como posibles causas, en términos hipotéticos, a la metodología docente o el nivel de exigencia, respectivamente.

A partir de estos antecedentes se puede afirmar que las características institucionales, potencialmente generadoras de estrés, no han sido lo suficientemente investigadas, sin embargo, algunos autores como Amigo (2000) y Cruz y Vargas (2001) hacen referencia al concepto impredecibilidad, como rasgo central que deben tener las situaciones para ser generadoras de estrés. Por su parte Barraza (2010), a través del constructo instituciones altamente demandantes, construye una tipología de características que poseen las instituciones que son potencialmente generadoras de estrés. Esta tipología se basa en seis características que definen los tipos de entornos institucionales por el tipo y/o la cantidad de las demandas que presentan:

- Entornos exageradamente demandantes

- Entornos con demandas novedosas

- Entornos altamente impredecibles en las demandas

- Entornos escasamente controlables en sus demandas

- Entornos con demandas de alta duración

- Entornos con demandas de elevada intensidad.

A partir de esta tipología se diseñó el Inventario de Características Institucionales Estresoras (Barraza, Ortega \& Martínez, 2010) que reportó una confiabilidad en alfa de Cronbach de .93 y una confiabilidad por mitades según la fórmula de Spearman-Brown de .87. Así mismo, se obtuvieron evidencias de validez, basadas en la estructura interna, a partir de los procedimientos denominados validez de consistencia interna y análisis de grupos contrastados, lo que permitió reconocer que: a) todos los ítems correlacionaban positivamente (con un nivel de significación de .00) con el puntaje global obtenido por cada encuestado, y b) todos los ítems permitían discriminar (con un nivel de significación de .00) entre los grupos que reportaban un alto y bajo nivel de las características institucionales generadoras de estrés en la institución.

No obstante, esta perspectiva teórica y el inventario diseñado a partir de ella representan un avance en la búsqueda de las características contextuales/ 
situacionales que coadyuven en la explicación de la generación del estrés, es necesario reconocer varias limitaciones al respecto: el inventario fue aplicado solamente a 73 alumnos de postgrado, no se realizó un análisis factorial para conocer su estructura interna, y sobre todo, no se reporta que cada uno de los ítems, de las dimensiones, establecidas teóricamente, o el inventario, en lo general, hayan sido sometidos a correlación con el estrés para determinar si realmente existía una relación. A partir de estas limitaciones se plantea como objetivo de la presente investigación la validación psicométrica del Inventario de Características Institucionales Estresoras

\section{MÉTODO}

\subsection{Diseño}

El presente estudio se considera de carácter instrumental (Montero \& León, 2005), ya que tiene por objetivo central determinar las propiedades psicométricas del instrumento en mención. De manera secundaria puede ser clasificado como transversal (la recolección de la información se realizó en un solo momento) y no experimental (no existió manipulación de variables).

\subsection{Participantes}

La presente investigación se realizó con una muestra de 300 alumnos de la Universidad Pedagógica de Durango, ubicada en la ciudad de Durango, en el estado de Durango, en México. La selección de los elementos muestrales fue no probabilística, de tipo intencional y determinada por la accesibilidad a los mismos. Como la aplicación del cuestionario se realizó en las propias aulas escolares de los alumnos, y en días de clases, los criterios de inclusión fueron solamente ser alumno de esa institución, haber asistido ese día a clases y tener la disposición para responderlo.

La distribución de los alumnos encuestados, según las variables sociodemográficas solicitadas, fue la siguiente:

- El $32 \%$ es del sexo masculino y el $68 \%$ del sexo femenino.

- La edad mínima es de 19 años y la máxima de 67, siendo el promedio 29 años de edad, con una desviación estándar de 11,16.

- El $75 \%$ cursaba en ese momento la Licenciatura y el $25 \%$ restante la Maestría o Doctorado.

\subsection{Instrumento}

Para la recolección de la información se utilizó el cuestionario denominado Inventario de Características Institucionales Estresoras (ICIE) (Barraza, 2009), el cual se compone de 25 ítems que se responden en un escalamiento tipo 
Likert de cuatro valores (Totalmente de Acuerdo, De Acuerdo, En Desacuerdo y Totalmente en Desacuerdo): Estos ítems se agrupan en seis dimensiones:

1. Entornos altamente demandantes (D): $1,6,10,13,18$

2. Entornos con demandas novedosas (DN): $3,7,9,12$,

3. Entornos altamente impredecibles en sus demandas (I): 11, 16, 19, 24

4. Entornos escasamente controlables en sus demandas (EC): 5, 15, 20, 23,

5. Entornos con demandas de alta duración (AD): 2, 8, 21, 25.

6. Entornos con demandas de elevada intensidad (IN): 4, 14, 17, 22

A este cuestionario se le agregaron tres ítems sobre datos sociodemográficos (sexo, edad y nivel de estudios) y un ítem donde se les pidió en una escala del uno al diez que señalaran el nivel de estrés que presentaban en ese momento.

\subsection{Procedimiento}

Para la aplicación del instrumento se contó con la aprobación de la autoridad correspondiente, en este caso el Director General de la Universidad Pedagógica de Durango. Para esto se le presentó el proyecto de la investigación y el aval del Comité de Investigación institucional; así mismo, se le explicó que no existía ningún riesgo para los participantes. Una vez obtenida la aprobación se procedió a aplicar el cuestionario de manera grupal. La selección de los grupos donde se aplicó fue determinada por su accesibilidad y el apoyo brindado por los maestros titulares del grupo. En la aplicación estuvo presente el propio investigador por lo que dio las orientaciones necesarias, explicó el carácter voluntario y confidencial de la participación y despejó dudas cuando las hubo.

Una vez aplicado se construyó la base de datos en el programa SPSS versión 23. Para evitar los valores perdidos, o tener la necesidad de recurrir a su imputación, fueron desechados 12 cuestionarios que mostraban valores perdidos y únicamente se capturaron en la base de datos aquellos que no presentaban ningún valor perdido. Por lo que la base de datos final se conformó por 300 participantes.

\subsection{Análisis de datos}

Una vez recolectada la información se analizó en seis etapas: a) en un primer momento se realizó el análisis filtro a partir de un análisis correlacional a través del coeficiente de correlación $r$ de Pearson entre cada uno de los ítems y el nivel de estrés autopercibido por los propios alumnos; a partir de este análisis se hizo una selección de ítems y el resto de los análisis se realizaron 
con la versión breve del inventario, b) posteriormente se obtuvo la estadística descriptiva, c) en un tercer momento se obtuvo la confiabilidad a través del estadístico alfa de Cronbach, d) posteriormente se realizó el análisis de consistencia interna a través del estadístico $r$ de Pearson, e) en un quinto momento se efectuó el Análisis Factorial Exploratorio, y f) finalmente, se realizó el Análisis Factorial Confirmatorio. Este último análisis se desarrolló con el programa LISREL 8.80 (Student).

\subsection{Consideraciones éticas}

El protocolo que dio origen a la presente investigación fue aprobado por el Comité de Investigación de la institución de adscripción del autor, con apego a la protección de los derechos y garantías de los participantes en la investigación, según la sección 8.01 de los principios éticos de la American Psychological Association (2017). Para asegurar la protección de los participantes se desarrollaron cuatro acciones: a) en el cuestionario no se les solicitó el nombre, número de lista u otro dato que pudiera comprometer su anonimato; b) en los primeros párrafos del instrumento, al presentar el cuestionario, se les aseguró la confidencialidad y el carácter general con que se analizarían los resultados; c) en la sección de datos personales del cuestionario solamente se solicitó información referente a las variables sociodemográficas que se utilizarían para la caracterización de la muestra y en ningún momento se les solicitó algún otro tipo de información que pudiera comprometer el anonimato de los participantes; y d) en el párrafo introductorio del cuestionario se les indicó que su participación era voluntaria por lo que estaban en total libertad de responderlo, o de no hacerlo. Con estas cuatro acciones se les brindó certeza a los participantes sobre la confidencialidad de los resultados y además se obtuvo, de manera indirecta, el consentimiento informado.

Así mismo, y de acuerdo al artículo 17, fracción I, del Reglamento de la Ley General de Salud en Materia de Investigación en Salud (Secretaría de Salud, 1987), se considera que este estudio puede ser catalogado como una investigación sin riesgo, ya que se utilizó para la recolección de la información solamente cuestionarios que no afectaron o modificaron de manera intencionada variables fisiológicas, psicológicas y/o sociales de los participantes.

\subsection{Conflicto de interés}

El autor declara que en el desarrollo de la presente investigación, así como en la difusión de sus resultados, no existe ningún conflicto de interés manifiesto o reconocido. 


\section{RESULTADOS}

Los resultados se presentan agrupados en los seis rubros que comprenden las etapas de análisis: análisis filtro, estadística descriptiva, confiabilidad, Análisis de Consistencia Interna, Análisis Factorial Exploratorio y Análisis Factorial Confirmatorio.

\subsection{Análisis filtro}

El primer paso del análisis era confirmar si, más allá de los fundamentos teóricos, cada ítem podría o no relacionarse de manera estadística con la presencia del estrés. Para determinar esto se realizó un análisis correccional cuyos resultados se muestran en la Tabla 1 . Como se puede observar solo diez ítems se correlacionan de manera positiva, y con un nivel de significación menor a .05, con el nivel de estrés autopercibido. Ante esto se decidió eliminar los 15 ítems que no se relacionan y dejar una versión breve del inventario con solo diez ítems y a partir de esta segunda versión realizar el resto de los análisis.

Tabla 1.

Valor $r$ de Pearson en el análisis efectuado entre cada uno de los ítems del ICIE y el nivel de estrés autopercibido

\begin{tabular}{|c|c|c|c|}
\hline DIM. & NÚM. & ÍTEMS & $r$ \\
\hline D & 1 & Nuestros maestros suelen encargar muchas tareas escolares para que las realicemos en nuestras casas & .125 \\
\hline$A D$ & 2 & $\begin{array}{l}\text { Durante todo el semestre nuestros maestros nos encargaron mucho trabajo, al grado que no había tiempo } \\
\text { para descansar }\end{array}$ & $.209^{* *}$ \\
\hline DN & 3 & $\begin{array}{l}\text { El tipo de trabajo que nos piden nuestros maestros es tan diferente a lo que hacía antes, que se me dificulta } \\
\text { hacerlo }\end{array}$ & .158 \\
\hline IN & 4 & Nuestros maestros quieren que en poco tiempo hagamos actividades o tareas muy complejas y difíciles & .149 \\
\hline EC & 5 & Nuestros maestros deciden la forma de evaluación sin tomarnos en cuenta & -.068 \\
\hline D & 6 & $\begin{array}{l}\text { Cada uno de nuestros maestros nos encarga mucho trabajo pensando que solo tenemos que hacer las activi- } \\
\text { dades referentes a su clase }\end{array}$ & $.180^{*}$ \\
\hline DN & 7 & La forma de evaluar en esta institución es diferente a la utilizada antes en otras instituciones & -.011 \\
\hline$A D$ & 8 & Todo el semestre me sentí agobiado(a) por tanta tarea que nos encargaban & $.225^{* *}$ \\
\hline DN & 9 & Los maestros nos exigen que hagamos trabajos que nunca antes habíamos hecho & $.262^{* *}$ \\
\hline D & 10 & Es tanta la tarea que me encargan que tengo que dedicar mi "tiempo libre" a hacerla & $.185^{*}$ \\
\hline । & 11 & $\begin{array}{c}\text { Nuestros maestros improvisan los trabajos que nos encargan, al inicio del semestre no nos dijeron lo que } \\
\text { íbamos a hacer }\end{array}$ & .123 \\
\hline DN & 12 & En esta institución hay muchas situaciones nuevas a las que no estaba acostumbrado(a). & $.201^{* *}$ \\
\hline D & 13 & Son tantas las tareas escolares que debo realizar, que es necesario que me desvele para lograrlo & $.211^{* *}$ \\
\hline IN & 14 & $\begin{array}{l}\text { Las actividades que nos encargan nuestros maestros son tan complejas y difíciles que necesitamos invertir } \\
\text { mucho tiempo para realizarlas }\end{array}$ & $.189^{*}$ \\
\hline
\end{tabular}




\begin{tabular}{|c|c|c|c|}
\hline DIM. & NÚM. & ÍTEMS & r \\
\hline EC & 15 & Únicamente nuestros maestros deciden el trabajo que tenemos que realizar & .078 \\
\hline I & 16 & $\begin{array}{l}\text { Nuestros maestros nunca nos dejan claro la forma de evaluar y hasta el último momento sabemos cómo lo van } \\
\qquad \text { a hacer }\end{array}$ & .091 \\
\hline IN & 17 & $\begin{array}{l}\text { Son tan complicados y difíciles los trabajos que tengo que entregar cada semestre, que "ya no encuentro la } \\
\text { salida". }\end{array}$ & $.270^{* *}$ \\
\hline D & 18 & $\begin{array}{l}\text { Los trabajos que nos encargan nuestros maestros son tan diferentes unos de otros que nos obligan a que } \\
\text { trabajemos más tiempo en ellos }\end{array}$ & $.226^{* *}$ \\
\hline I & 19 & Nuestros maestros encargan trabajos sin dejar claro cómo los quieren o qué es lo que van a evaluar en ellos & .045 \\
\hline EC & 20 & $\begin{array}{l}\text { Nuestros maestros deciden cuándo se deben entregar los trabajos que nos solicitan, sin tomar en cuenta el } \\
\text { tiempo del que disponemos para hacerlos }\end{array}$ & .029 \\
\hline$A D$ & 21 & Nuestros maestros, al terminar un trabajo, inmediatamente nos encargan otro & .115 \\
\hline IN & 22 & $\begin{array}{c}\text { El tipo de examen/evaluación que utilizan nuestros maestros es tan complejo y difícil, que nos obligan a que le } \\
\text { dediquemos más tiempo al estudio }\end{array}$ & .140 \\
\hline EC & 23 & En esta institución todo lo deciden nuestros maestros, los alumnos no tenemos ni voz, ni voto & -.038 \\
\hline I & 24 & $\begin{array}{l}\text { En esta institución cada maestro es tan diferente que no se puede saber con anterioridad cómo vamos a } \\
\text { trabajar o cómo nos van a evaluar }\end{array}$ & .091 \\
\hline$A D$ & 25 & $\begin{array}{c}\text { Esta institución se caracteriza porque los maestros encargan trabajos que requieren mucho tiempo para } \\
\text { realizarse }\end{array}$ & .048 \\
\hline
\end{tabular}

Nota: Dim. (Dimensión), D (Entornos altamente demandantes), DN (Entornos con demandas novedosas), I (Entornos altamente impredecibles en sus demandas), EC (Entornos escasamente controlables en sus demandas), AD (Entornos con demandas de alta duración), IN (Entornos con demandas de elevada intensidad). ${ }^{*} \mathrm{p}<.05$, una cola, en negritas. $* * p<.01$, una cola, en negritas.

\subsection{Estadística descriptiva}

La media aritmética y la desviación estándar de cada uno de los ítems de la versión breve del inventario se presentan en la Tabla 2. Como se puede observar las situaciones que se presentan con mayor frecuencia en la institución donde cursan sus estudios los encuestados son: "Cada uno de nuestros maestros nos encarga mucho trabajo pensando que solo tenemos que hacer las actividades referentes a su clase" y "En esta institución hay muchas situaciones nuevas a las que no estaba acostumbrado(a)"; mientras que las que menos se presentan son: "Son tan complicados y difíciles los trabajos que tengo que entregar cada semestre, que ya no encuentro la salida" y "Los trabajos que nos encargan nuestros maestros son tan diferentes unos de otros que nos obligan a que trabajemos más tiempo en ellos".

La media general de todo el cuestionario es de 1,57 (51\%), mientras que la media del nivel de estrés autopercibido es de 7,1 (71\%). Estos valores interpretados con un baremo indicativo de tres valores (de 0 a $33 \%$ leve, de $34 \%$ a $66 \%$ moderado, y de $67 \%$ a $100 \%$ profundo) permiten afirmar que las características institucionales generadoras de estrés se presentan con un nivel moderado, mientras que el estrés se presenta con un nivel profundo. 
Tabla 2.

Estadística descriptiva de los ítems que conforman la versión breve del ICIE

\begin{tabular}{|c|c|c|c|}
\hline NÚM. & ÍTEMS & $\mu$ & $\sigma$ \\
\hline 2 & $\begin{array}{c}\text { Durante todo el semestre nuestros maestros nos encargaron mucho trabajo, al grado que no había tiempo para } \\
\text { descansar }\end{array}$ & 1.50 & .792 \\
\hline 6 & $\begin{array}{l}\text { Cada uno de nuestros maestros nos encarga mucho trabajo pensando que solo tenemos que hacer las activi- } \\
\text { dades referentes a su clase }\end{array}$ & 1.84 & .778 \\
\hline 8 & Todo el semestre me sentí agobiado(a) por tanta tarea que nos encargaban & 1.81 & .766 \\
\hline 9 & Los maestros nos exigen que hagamos trabajos que nunca antes habíamos hecho & 1.51 & .740 \\
\hline 10 & Es tanta la tarea que me encargan que tengo que dedicar mi "tiempo libre" a hacerla & 1.51 & .712 \\
\hline 12 & En esta institución hay muchas situaciones nuevas a las que no estaba acostumbrado(a). & 1.70 & .825 \\
\hline 13 & Son tantas las tareas escolares que debo realizar, que es necesario que me desvele para lograrlo & 1.63 & .781 \\
\hline 14 & $\begin{array}{l}\text { Las actividades que nos encargan nuestros maestros son tan complejas y difíciles que necesitamos invertir mucho } \\
\text { tiempo para realizarlas }\end{array}$ & 1.59 & .744 \\
\hline 17 & Son tan complicados y difíiles los trabajos que tengo que entregar cada semestre, que "ya no encuentro la salida". & 1.24 & .739 \\
\hline 18 & $\begin{array}{l}\text { Los trabajos que nos encargan nuestros maestros son tan diferentes unos de otros que nos obligan a que trabaje- } \\
\text { mos más tiempo en ellos }\end{array}$ & 1.41 & .697 \\
\hline
\end{tabular}

Nota: se destacan con negritas las medias más altas y con cursiva las más bajas. Se respeta la numeración original de los ítems.

\subsection{Confiabilidad}

Como tercer momento se efectuó el análisis de confiabilidad obteniendo un coeficiente de .71. Como complemento se realizó el análisis de confiabilidad en caso de eliminar un ítem y los resultados mostraron que, en todos los casos, el coeficiente de confiabilidad baja.

\subsection{Análisis de Consistencia interna}

Los resultados del análisis de consistencia interna se muestran en la Tabla 3 y como se puede observar todos los ítems se correlacionan de manera positiva, con un nivel de significación menor a .01, con la media general.

Tabla 3.

Valor $r$ de Pearson en el análisis efectuado entre cada uno de los ítems del ICIE y la media general de cada cuestionario

\begin{tabular}{crc}
\hline NÚM. & ÍTEMS & r \\
\hline 2 & Durante todo el semestre nuestros maestros nos encargaron mucho trabajo, al grado que no había tiempo para descansar & $.501^{* *}$ \\
6 & Cada uno de nuestros maestros nos encarga mucho trabajo pensando que solo tenemos que hacer las actividades referentes a su clase & $.574^{* *}$ \\
8 & Todo el semestre me sentí agobiado(a) por tanta tarea que nos encargaban & $.432^{* *}$ \\
9 & Los maestros nos exigen que hagamos trabajos que nunca antes habíamos hecho & $.578^{* *}$ \\
10 & Es tanta la tarea que me encargan que tengo que dedicar mi "tiempo libre" a hacerla & $.545^{* *}$ \\
12 & En esta institución hay muchas situaciones nuevas a las que no estaba acostumbrado(a). & $.564^{* *}$ \\
13 & Son tantas las tareas escolares que debo realizar, que es necesario que me desvele para lograrlo & $.505^{* *}$ \\
14 & Las actividades que nos encargan nuestros maestros son tan complejas y difíiles que necesitamos invertir mucho tiempo para realizarlas & $.573^{* *}$ \\
17 & Son tan complicados y difíciles los trabajos que tengo que entregar cada semestre, que "ya no encuentro la salida". & $.571^{* *}$ \\
18 & Los trabajos que nos encargan nuestros maestros son tan diferentes unos de otros que nos obligan a que trabajemos & $.463^{* *}$ \\
& & más tiempo en ellos \\
\hline
\end{tabular}

${ }^{* *} \mathrm{p}<.01$, una cola. Se respeta la numeración original de los ítems 


\subsection{Análisis Factorial Exploratorio}

Para el análisis factorial exploratorio se consideraron algunos criterios previos que permitieron valorar su viabilidad: la prueba de esfericidad de Bartlett fue significativa en .000 , y el test KMO de adecuación de la muestra alcanzó un valor de .805 , por lo que se consideró pertinente continuar con el análisis (Visauta \& Martori, 2005). En seguida se hizo una estimación de las comunalidades de los ítems que integran la versión breve del inventario; sus resultados se presentan en la Tabla 4. Para efecto de este análisis, se tomaron como ítems aceptables los de las comunalidades superiores a .3 (Pardo \& Ruiz, 2002) que, como se puede observar, fueron solamente ocho.

Tabla 4.

Comunalidades por ítems del ICIE

\begin{tabular}{|c|c|c|c|}
\hline NÚM. & ÍTEMS & INICIAL & EXTRACCIÓN \\
\hline 2 & $\begin{array}{c}\text { Durante todo el semestre nuestros maestros nos encargaron mucho trabajo, al grado que no había } \\
\text { tiempo para descansar }\end{array}$ & 1.000 & .545 \\
\hline 6 & $\begin{array}{l}\text { Cada uno de nuestros maestros nos encarga mucho trabajo pensando que solo tenemos que hacer las } \\
\text { actividades referentes a su clase }\end{array}$ & 1.000 & .557 \\
\hline 8 & Todo el semestre me sentí agobiado(a) por tanta tarea que nos encargaban & 1.000 & .182 \\
\hline 9 & Los maestros nos exigen que hagamos trabajos que nunca antes habíamos hecho & 1.000 & .403 \\
\hline 10 & Es tanta la tarea que me encargan que tengo que dedicar mi "tiempo libre" a hacerla & 1.000 & .444 \\
\hline 12 & En esta institución hay muchas situaciones nuevas a las que no estaba acostumbrado(a). & 1.000 & .356 \\
\hline 13 & Son tantas las tareas escolares que debo realizar, que es necesario que me desvele para lograrlo & 1.000 & .238 \\
\hline 14 & $\begin{array}{l}\text { Las actividades que nos encargan nuestros maestros son tan complejas y difíciles que necesitamos } \\
\text { invertir mucho tiempo para realizarlas }\end{array}$ & 1.000 & .426 \\
\hline 17 & $\begin{array}{c}\text { Son tan complicados y difíciles los trabajos que tengo que entregar cada semestre, que "ya no encuen- } \\
\text { tro la salida". }\end{array}$ & 1.000 & .473 \\
\hline 18 & $\begin{array}{l}\text { Los trabajos que nos encargan nuestros maestros son tan diferentes unos de otros que nos obligan a } \\
\qquad \text { que trabajemos más tiempo en ellos }\end{array}$ & 1.000 & .408 \\
\hline
\end{tabular}

Nota: valores superiores a .3 en negritas. Se respeta la numeración original de los ítems.

Por su parte, en el análisis factorial, se usó el método de componentes principales con rotación Varimax que posibilitó identificar dos componentes que explican $40 \%$ de la varianza total; en la Tabla 5 se presentan los dos factores y la saturación de cada uno de los ítems ubicados en cada factor y como se puede observar todos los ítems pudieron ubicarse en alguno de los dos factores al tener saturaciones mayores a .35 (Moral de la Rubia, 2006).

El primer componente puede ser denominado características de las actividades escolares, ya que hace referencia a aquellas características como novedad, complejidad, dificultad, etc. de las diferentes actividades solicitadas por los docentes; mientras que el segundo componente, que puede ser denominado cantidad de trabajo escolar, hace referencia al gran número de actividades escolares que los profesores les exigen a sus alumnos que 
realicen. Cabe destacar que se presentó el caso del ítem 13 (numeración original) que conceptualmente corresponde al segundo componente pero satura en el primero.

Tabla 5.

Matriz factorial de los ítems que conforman la versión breve del ICIE

\begin{tabular}{|c|c|c|c|}
\hline \multirow{2}{*}{ NÚM. } & \multirow{2}{*}{ ÍTEMS } & \multicolumn{2}{|c|}{ COMPONENTE } \\
\hline & & 1 & 2 \\
\hline 2 & $\begin{array}{l}\text { Durante todo el semestre nuestros maestros nos encargaron mucho trabajo, al grado que no había } \\
\text { tiempo para descansar }\end{array}$ & & .738 \\
\hline 6 & $\begin{array}{l}\text { Cada uno de nuestros maestros nos encarga mucho trabajo pensando que solo tenemos que hacer las } \\
\text { actividades referentes a su clase }\end{array}$ & & .732 \\
\hline 8 & Todo el semestre me sentí agobiado(a) por tanta tarea que nos encargaban & & .395 \\
\hline 9 & Los maestros nos exigen que hagamos trabajos que nunca antes habíamos hecho & .583 & \\
\hline 10 & Es tanta la tarea que me encargan que tengo que dedicar mi "tiempo libre" a hacerla & & .631 \\
\hline 12 & En esta institución hay muchas situaciones nuevas a las que no estaba acostumbrado(a). & .559 & \\
\hline 13 & Son tantas las tareas escolares que debo realizar, que es necesario que me desvele para lograrlo & .431 & \\
\hline 14 & $\begin{array}{l}\text { Las actividades que nos encargan nuestros maestros son tan complejas y difíciles que necesitamos inver- } \\
\qquad \text { tir mucho tiempo para realizarlas }\end{array}$ & .627 & \\
\hline 17 & $\begin{array}{l}\text { Son tan complicados y difíciles los trabajos que tengo que entregar cada semestre, que "ya no encuentro } \\
\text { la salida". }\end{array}$ & .679 & \\
\hline 18 & $\begin{array}{c}\text { Los trabajos que nos encargan nuestros maestros son tan diferentes unos de otros que nos obligan a que } \\
\text { trabajemos más tiempo en ellos }\end{array}$ & .635 & \\
\hline
\end{tabular}

Nota: Se respeta la numeración original de los ítems.

\subsection{Análisis Factorial Confirmatorio}

El Análisis Factorial Confirmatorio se decidió realizarlo con dos modelos: el primero era tal como fue reportado por el Análisis Factorial Exploratorio con dos factores: el factor uno con seis ítems y el factor dos con cuatro ítems; el segundo era haciendo la corrección del ítem 13 (numeración original) ubicándolo en el factor dos de tal manera que los dos factores quedaban con cinco ítems.

Los resultados del Análisis Factorial Confirmatorio se presentan en la Tabla 6. Como se puede observar no hay una diferencia significativa en los dos modelos y solamente el valor de $\chi 2$ es menor en el primer modelo; en ambos casos presentan un valor pequeño de chi cuadrada (el resultado de $\mathbf{\chi} \mathbf{2}$ / df en ambos casos es menor a uno) y el valor RMSEA es inferior al .05, lo que de acuerdo a Hu y Bentler (1995) se puede considerar óptimo. 
Tabla 6.

Valores obtenidos en el Análisis Factorial Confirmatorio en los dos modelos de la versión breve del ICIE

\begin{tabular}{ccccccc}
\hline $\begin{array}{c}\text { VERSIÓN DEL } \\
\text { INVENTARIO }\end{array}$ & $\mathbf{X}^{\mathbf{2}}$ & df & P-VALUE & RMSEA & $\begin{array}{c}\text { CONFIDENCE INTERVAL } \\
\text { FOR RMSEA }\end{array}$ & $\begin{array}{c}\text { P-VALUE FOR TEST OF } \\
\text { CLOSE FIT }\end{array}$ \\
\hline $\begin{array}{c}\text { Versión de } 10 \text { ítems respe- } \\
\text { tando la ubicación del ítem } \\
\text { trece según el AFE }\end{array}$ & 16,70 & 34 & 0,99439 & 0,000 & $0,00-0,00$ & $\begin{array}{c}\text { (RMSEA }<.05) \\
=1.00\end{array}$ \\
$\begin{array}{c}\text { Versión de } 10 \text { ítems acomo- } \\
\text { dando el ítem trece en el } \\
\text { factor 2 }\end{array}$ & 19,47 & 34 & 0,97823 & 0,000 & $0,00-0,00$ & (RMSEA $<.05)$ \\
& & & & & & 1.00 \\
\hline
\end{tabular}

\section{DISCUSIÓN}

La vida cotidiana de un alumno está llena de experiencias escolares que le son potencialmente estresantes. Aunque estas situaciones no tienen el mismo peso de los acontecimientos vitales, la investigación al respecto ha indicado que el estrés puede llegar a afectar la salud física y mental de las personas (Lazarus, 2000; Pineda-Roa \& Navarro-Segura, 2019).

Para avanzar en el conocimiento de este tipo de estrés es necesario identificar las variaciones de la personalidad y las características de las condiciones ambientales que generan el significado relacional que conduce al estrés. Las variaciones o rasgos de personalidad han sido mayoritariamente estudiados, mientras que las características de las condiciones ambientales no lo han sido. En este sentido, cuando Lazarus (2000) hace un recuento de ambos aspectos queda de manifiesto la precisión teórica y evidencia empírica que respalda el estudio de las variaciones o rasgos de personalidad, mientras que al abordar las condiciones ambientales hace referencia a cuatro aspectos: demandas sociales, limitaciones, oportunidad y cultura, empero tres de ellos se presentan de manera sucinta, sin posicionamientos teóricos o investigaciones que lo respalden y el cuarto de ellos, a pesar de la evidencia empírica mencionada queda la sensación de ser una causa distal en la generación del estrés. Por su parte Moos $(1995,2010)$ hace referencia a situaciones ambientales de carácter psicosocial que se alejan de las características situacionales que son las de interés para la presente investigación.

Ante esta situación cobra relevancia el interés de avanzar en la investigación que permita reconocer las características particulares de los contextos que determinan las condiciones situacionales que entran en transacción con los rasgos de personalidad de los sujetos para generar el significado relacional 
que genera el estrés. Paralelo a los posicionamientos teóricos al respecto, cobra importancia revisar los instrumentos elaborados para tal fin.

En el caso de la presente investigación se decidió evaluar las propiedades psicométricas del ICIE para determinar si realmente constituye una opción válida para reconocer las características del contexto escolar que pueden constituirse en condiciones situacionales que entran en el proceso de transacción que genera el estrés. El primer análisis efectuado, a nivel de filtro, permitió reconocer que no todos los ítems se relacionaban con el estrés por lo que se decidió hacer una versión breve de este inventario y a partir de ella analizar sus propiedades psicométricas. A partir del análisis efectuado se puede afirmar que la versión breve del ICIE presenta las siguientes propiedades psicométricas:

a. Un nivel de confiabilidad de .71 en alfa de cronbach, el cual puede ser considerado como respetable, según la escala de valores propuesta por George y Mallery (2003).

b. En el análisis de consistencia interna, realizado a partir del procedimiento establecido por Salkind (1999), los resultados mostraron que todos los ítems correlacionaron positivamente (con un nivel de significación de .00) con el puntaje global obtenido por cada encuestado. En ese sentido, se confirma la homogeneidad del instrumento lo que conduce a reconocer que todos los ítems miden lo mismo, o en otras palabras, representan el mismo dominio empírico de referencia, y tienen una sola direccionalidad.

c. El análisis factorial, tanto exploratorio como confirmatorio, permitió identificar dos dimensiones de la versión breve del inventario: 1) características de las actividades escolares, y 2 ) cantidad de trabajo escolar.

La dimensión "características de las actividades escolares" agrupa ítems que tienen que ver con los factores situacionales que influyen en la valoración, en términos de Lazarus y Folkman (1986), y características de las dimensiones, 2,3, 4 y 5 que indagaba originalmente el Inventario de Características Institucionales Estresoras; por su parte, la dimensión "cantidad de trabajo escolar" hace referencia a la frecuencia e intensidad de las demandas que son características presentes en las dimensiones 1 y 6 que indagaba originalmente el Inventario de Características Institucionales Estresoras.

Las dos dimensiones del instrumento están asociadas a estresores identificados plenamente en la literatura sobre el tema, p. ej. el Inventario de 
Estresores Académicos, utilizado por Barraza y León (2015), el Inventario de Estrés Académico de Mazo, Gutiérrez y Londoño (2009, citados en Mazo, Londoño \& Gutiérrez, 2013) y la Escala de Estresores Académicos, utilizada por Cabanach, Souto-Gestal y Franco (2016). En el caso de la literatura en lengua inglesa suelen aparecer normalmente solo estresores relacionados a la dimensión "cantidad de trabajo escolar" como es el caso de Educational Stress Scale for Adolescents (Sun, Dunne, Hou \& Xu, 2011), Academic Stress Scale (Xiao, 2013) y College Stress Inventory (Scott, Bradford, Villarreal \& Kavanagh, 1993).

Cabe destacar que el instrumento validado en la presente investigación no hace referencia única a lo que estresa, ya que esto lo convertiría en un inventario de estresores, sino a la posible causa, esto es, a las prácticas docentes o características de la actividad que generan ese estresor; dos ejemplos bastan para ilustrar esta situación: a) en el ítem "Los maestros nos exigen que hagamos trabajos que nunca antes habíamos hecho", no solo se hace referencia a la demanda de trabajo, sino a su característica central "la novedad", b) en el ítem "Cada uno de nuestros maestros nos encarga mucho trabajo pensando que solo tenemos que hacer las actividades referentes a su clase" no solo se hace referencia a la excesiva demanda de trabajo, sino a lo que lo origina "la focalización del maestro solamente en su asignatura".

Esta versión breve del ICIE presenta propiedades psicométricas adecuadas que la vuelve una opción válida para el estudio de las características potencialmente generadoras de estrés de las instituciones educativas; cabe resaltar que el interés en las variables contextuales es situacional y psicosocial por lo que se coincide con Barraza, et al. (2010) cuando afirman que el entorno es "el conjunto de situaciones, que de carácter normativo o contingente, definen y estructuran las relaciones de los diferentes agentes involucrados en un espacio organizacional delimitado" (p.7).

El demostrar que las propiedades psicométricas de esta versión breve del ICIE son adecuadas para la indagación empírica permite impulsar el estudio de la perspectiva contextualista del estrés (Aldwin \& Revenson, 1987; Lazarus \& Folkman, 1986) que, como ya se mencionó anteriormente, pretende brindar un papel más protagónico a la naturaleza de la situación, en el entendido de que en el estrés intervienen tanto variables individuales como ambientales (García \& Vélez, 2018). 
A manera de cierre es necesario mencionar que la principal limitación del presente estudio es que la muestra de alumnos corresponde a una sola institución educativa por lo que ante el riesgo de tener una carácter eminentemente idiosincrático se debe hacer una réplica del trabajo tomando como base alumnos de diferentes instituciones de educación superior, así como de educación media superior, que en esta investigación no fueron considerados. Así mismo, es necesario revisar en las próximas aplicaciones del instrumento la ubicación del ítem siete, ya que en el Análisis Factorial Exploratorio satura en un factor diferente a su carga conceptual, mientras que en el Análisis Factorial Confirmatorio demostró que puede estar en uno $\mathrm{u}$ otro factor; por el momento se ha decidido ubicarlo donde corresponde conceptualmente que sería el factor que hace referencia a la cantidad de trabajo.

Agradecimiento: Se agradece a las autoridades de la Universidad Pedagógica de Durango por el apoyo brindado para la realización de la presente investigación; amablemente permitieron la aplicación de los instrumentos en sus aulas escolares y le otorgaron el tiempo necesario al investigador para el desarrollo de la investigación.

Financiamiento: La investigación se realizó sin financiamiento directo. Solamente la institución de adscripción del autor brindó las facilidades laborales para su realización.

\section{REFERENCIAS}

Aldwin, C. M. \& Revenson, T. A. (1987). Does coping help? A reexamination of the relation between coping and mental health. Journal of Personality and Social Psychology, 53(2), 337-348. Recovered from https://www.researchgate.net/ profile/Carolyn_Aldwin/publication/19535402_Does_Coping_Help_A_Reexamination_of_the_Relation_Between_Coping_and_Mental_Health/links/00b7d$52 f 27 \mathrm{fcb} 2 \mathrm{c} 209000000 / D o e s-C o p i n g-H e l p-A-R e e x a m i n a t i o n-o f-t h e-R e l a t i o n-B e-$ tween-Coping-and-Mental-Health.pdf

Álvarez, L.A., Gallegos, R.M., \& Herrera, P.S. (2018). Estrés académico en estudiantes de tecnología superior. Universitas. Revista de Ciencias Sociales y Humanas, 28, 193-209. https://doi.org/10.17163/uni.n28.2018.10

American Psychological Association (2017). Ethical principles of psychologists and code of conduct. http://www.apa.org/ethics/code/.

Andretta, J.R., McKay, M.T., \& Byrne, D.G. (2018). Psychometric properties of the adolescent stress questionnaire-short form scores and association with self-efficacy. Journal of Psychiatry and Behavioral Sciences, 2, 1-6. https:// meddocsonline.org/journal-of-psychiatry-and-behavioral-sciences/psychometric-properties-of-the-adolescent-stress-questionnaire-short-form-scores-and-association-with-self-efficacy.pdf

Anjali, S. (2018). Procrastination, stress and academic achievement among the B. Ed. Students. Educational Quest- An International Journal of Education and Applied Social Sciences, 9(1), 125-129. https://doi.org/10.30954/2230-7311.2018.04.17

Amigo V. I. (2000). El precio biológico de la civilización. Madrid, España: Celeste Ediciones S.A. 
Arce, R., Fariña, F., Novo, M. \& Seijo, D. (2012). Efecto del sistema de enseñanza en el rendimiento académico, burnout experimentado y estrés académico. Aula Abierta, 40(2), 3-10. https://dialnet.unirioja.es/ejemplar/302897

Barker, E.T., Howard, A.L., Villemaire-Krajden, R., \& Galambos, N.L. (2018). The rise and fall of depressive symptoms and academic stress in two samples of university students. Journal of Youth and Adolescence, 47(6), 1252-1266. https://doi. org/10.1007/s10964-018-0822-9

Barraza, A. (2009). Inventario de Características Institucionales Estresoras (ICIE). Investigación Educativa Duranguense, 5(11), 56-58. http://www.upd.edu.mx/ $\mathrm{PDF} /$ Revistas/InvestigacionEducativaDuranguense11.pdf

Barraza, A. (2010). La relación persona-entorno como fuente generadora de estrés académico. Revista Internacional de Psicología, 11(1), 1-11. http://www.revistapsicologia.org/index.php/revista/article/view/59/56

Barraza, A. \& León, G. (2015). Estresores académicos en estudiantes de educación. Un estudio comparativo entre educandos mexicanos y costarricenses. Revista Electrónica Formación y Calidad Educativa (REFCaIE), 3(1), 69-92. http://runachayecuador.com/refcale/index.php/refcale/article/view/97/60http://runachayecuador.com/refcale/index.php/refcale/article/view/97/60

Barraza, A., Ortega, F. \& Martínez, L. M. (2010). Características institucionales generadoras de estrés académico. Un estudio exploratorio. Visión Educativa IUNAES, 4(9), 5-17. http://iunaes.mx/wp-content/uploads/2013/04/09-Abril-de-2010.pdf

Cabanach, R.G., Souto-Gestal, A. \& Franco, V. (2016). Escala de Estresores Académicos para la evaluación de los estresores académicos en estudiantes universitarios. Revista Iberoamericana de Psicología y Salud, 7(2), 41-50. https://doi. org/10.1016/j.rips.2016.05.001

Cruz, M. C. \& Vargas, F. L. (2001). Estrés, entenderlo es manejarlo. México: Alfa Omega.

Emiro, J., Amador, O., Calderon, G., Castañeda, T., Osorio, Y., \& Diez, P. (2018). Depresión y su relación con el consumo de sustancias psicoactivas, el estrés académico y la ideación suicida en estudiantes universitarios colombianos. Health and Addictions, 18(2), 227-239. https://www.researchgate.net/publication/326736876_Depresion_y_su_relacion_con_el_consumo_de_sustancias_ psicoactivas_el_estres_academico_y_la_ideacion_suicida_en_estudiantes_ universitarios_colombianos

Fernández de Castro, J., \& Luévano, E. (2018). Influencia del estrés académico sobre el rendimiento escolar en educación media superior. Revista Panaméricana de Pedagogía, 26, 97-117. http://portalderevistasdelaup.mx/revistapedagogia/ index.php/pedagogia/article/view/284/287

García, M., \& Vélez, H. (2018). Exploración de las características psicométricas del inventario de estrés ocupacional - OSI para el contexto colombiano. Psicogente, 21(39), 140-161. http://doi.org/10.17081/psico.21.39.2828

George, D. \& Mallery, P. (2003). SPSS for Windows step by step: A simple guide and reference. 11.0 update. Boston: Allyn \& Bacon.

González, M. P. (2008). Percepción de estrés, experiencias académicas estresantes y afrontamiento en los estudios. Una investigación comparativa entre estudiantes de educación de Perú y Suecia. Educación, XVII (32), 49-66. http://revistas.pucp. edu.pe/index.php/educacion/article/view/1799/1739

González, R., Souto-Gestal, A., \& Fernández, R. (2017). Perfiles de regulación emocional y estrés académico en estudiantes de fisioterapia. European Journal of Education and Psychology, 10(2), 57-67. https://doi.org/10.1016/j.ejeps.2017.07.002

González, R., Franco, V., Souto-Gestal, A., \& González, L. (2017). ¿Media la orientación de las metas académicas el estrés en estudiantes universitarios? Revista de 
Investigación en Educación, 15(2), 109-121. https://dialnet.unirioja.es/servlet/ articulo?codigo $=6175270$

Hamizah, N., Alavi, M., Abolghasem, S., \& Ahmadi, A. (2018). Academic stress and self-regulation among university students in Malaysia: mediator role of mindfulness. Behavioral Sciences, 8(1), 1-9. https://doi.org/10.3390/bs8010012

Harajyoti, M., Dipankar, G., Lipika, B. \& Nabanita, H. (2012). A Comparative study on stress and its contributing factors among the Graduate and Post-graduate students. Advances in Applied Science Research, 3(1), 399-406. http://www. imedpub.com/articles/a-comparative-study-on-stress-and-its-contributing-factors-among-thegraduate-and-postgraduate-students.pdf

Hu, L.T. \& Bentler, P.M. (1995). Fit indices in covariance structure modeling: Sensitivity to underparameterized model misspecification. Psychological Methods, 4(3), 424-453. https://pdfs.semanticscholar.org/a92c/9726361d9c1d165dbf2ea781b6c48363a816.pdf

Lazarus, R. S. \& Folkman, S. (1986). Estrés y procesos cognitivos. Barcelona, España: Martínez Roca.

Lazarus, R. S. (2000). Estrés y emoción. Manejo e implicaciones en nuestra salud. Bilbao, España: Desclée de Brouwer.

Macbani, P., Rubalcava, J.C., Vázquez, P., Ramírez, A., González, K., Arredondo, K. \& Trego, A. (2018). Estrés académico, estresores y afrontamiento en estudiantes de Odontología en el Centro de Estudios Universitarios Metropolitano Hidalgo [CEUMH]. Journal of Negative and No Positive Results: JONNPR, 3(7), 522-530. https://doi.org/10.19230/jonnpr.2512

Mazo, R., Londoño, K., \& Gutiérrez, Y.F. (2013). Estrés académico en estudiantes universitarios. Informes Psicológicos, 13(2), 121-134. https://revistas.upb.edu. co/index.php/informespsicologicos/article/view/2796/2441

Montero, I. \& León, O. (2005). Sistema de clasificación del método en los informes de investigación en psicología. Internacional Journal of Clinical and Health Psychology, 5(1), 115-127. http://www.redalyc.org/articulo.oa?id=33701007

Moos, R.H. (1995). Development and applications of new measures of life stressors, social resources, and coping reponses. European Journal of Psychological Assesment, 11(1), 1-13. https://doi.org/10.1027/1015-5759.11.1.1.

Moos, R.H. (2010). CRI-A Inventario de Respuestas de Afrontamiento-Adultos. Madrid: TEA.

Moos, R.H. (2018). Evaluating treatment environments. The quality of psychiatric and substance abuse programs. New York: Routledge. https://doi. org/10.4324/9781351291804

Moral de la Rubia, J. (2006). Análisis factorial exploratorio y aplicación al desarrollo de escalas. En R. Landeros \& M. González (comp.), Estadística con SPSS y metodología de la investigación (pp. 445-528). México: Trillas.

Pardo, A., \& Ruiz, M.A. (2002). SPSS 11. Guía para el análisis de datos. Madrid, España: McGraw-Hill.

Pineda-Roa, C.A., \& Navarro-Segura, M.C. (2019). Validación de una prueba para medir eventos vitales estresantes en adultos gay, lesbianas y bisexuales colombianos. Psicogente 22(41), 1-19. https://doi.org/10.17081/psico.22.41.3305

Quito, J.V., Tamayo, M.C., Buñay, D.P., \& Neira, O.S. (2017). Estrés académico en estudiantes de tercero de bachillerato de unidades educativas particulares del Ecuador. Revista Electrónica de Psicología Iztacala. 20(3), 253- 276. http://www. medigraphic.com/pdfs/epsicologia/epi-2017/epi173o.pdf

Ribes, E. (1990). Psicología y salud: Un análisis conceptual. Barcelona: Martínez Roca.

Rodríguez, B., Arenal, T., Vega, M.C., \& Blanco, L.E. (2018). Prevalencia de estresores académicos en estudiantes de Grado en Enfermería. Metas de Enfermería, 21(4), 
49-54. https://www.enfermeria21.com/revistas/metas/articulo/81233/prevalencia-de-estresores-academicos-en-estudiantes-de-grado-en-enfermeria/

Sakellari, E., Psychogiou, M., Georgiou, A., Papanidi, M., Vlachou, V., \& Sapountzi-Krepia, D. (2017). Exploring religiosity, self-esteem, stress, and depression among students of a cypriot university. Journal of Religion and Health, 57(1), 136-145. https://doi.org/10.1007/s10943-017-0410-4

Salkind, N.J. (1999). Métodos de Investigación. México: Prentice Hall.

Scott, V., Bradford, J., Villarreal, P., \& Kavanagh, J. (1993). Development of the college stress inventory for use with hispanic populations: a confinnatory analytic approach. Hispanic Journal of Behavioral Sciences, 15(4), 490-497. https://doi. org/10.1177/07399863930154004

Secretaría de Salud (1987). Reglamento de la Ley General de Salud en Materia de Investigación para la Salud. México: Porrúa.

Shashank P. B., Richa, Y. \& Prakash, B. B. (2011). A comparative study of stress among students of medicine, engineering, and nursing. Indian Journal Psychological Medicine, 33(2), 145-148. https://doi.org/10.4103/0253-7176.92064

Sun, J., Dunne, M.P., Hou, X. \& Xu, A. (2011). Educational stress scale for adolescents: development, validity, and reliability with Chinesestudents.JournalofPsychoeducational Assessment, 29(6), 534-546. https://doi.org/10.1177/0734282910394976

Velásquez-Muñoz, A., Cornejo-Aravena, C., Bustamante-Toncio, F., \& Mella-Guzmán, M. (2018). Estresores que influyen desfavorablemente en estudiantes chilenos de Obstetricia y Puericultura, durante las prácticas clínicas. EDUMECENTRO, 10(3), 12-24. http://scielo.sld.cu/scielo.php?pid=S2077-28742018000300002\&scrip$\mathrm{t}=$ sci_arttext\&tlng=pt

Vidal, J., Muntaner, A., \& Palau, P. (2018). Diferencias de estrés y afrontamiento del mismo según el género y cómo afecta al rendimiento académico en estudiantes universitarios. Contextos educativos: Revista de Educación, 22, 181-195. http:// doi.org/10.18172/con.3369

Visauta, B., \& Martori, J.C. (2005). Análisis estadístico con SPSS para Windows. México: McGraw-Hill.

Won, J. (2018). Testing the three-way interaction effect of academic stress, academic self-efficacy, and task value on persistence in learning among Korean college students. Higher Education, 76(5), 921-935. https://doi.org/10.1007/s10734018-0255-0

Xiao, J. (2013). Academic Stress, Test Anxiety, and Performance in a Chinese High School Sample: The Moderating Effects of Coping Strategies and Perceived Social Support. (Dissertation). Georgia State University. http://scholarworks.gsu.edu/ cgi/viewcontent.cgi?article=1093\&context=cps_diss

You-Wei, Y., Rong-Mao, L., Yan-Kui, S., \& Ming-Yan, L. (2018). The relationship between adolescent academic stress and sleep quality: A multiple mediation model. Social Behavior and Personality: an International Journal, 46(1), 63-77. https://doi.org/10.2224/sbp.6530

\section{(cc) BY}


